\title{
Infinitely Many Trees with Maximum Number of Holes Zero, One, and Two
}

\author{
Srinivasa Rao Kola $\mathbb{D}^{\text {, }}$, Balakrishna Gudla, and P. K. Niranjan \\ Department of Mathematical and Computational Sciences, National Institute of Technology Karnataka, Surathkal, India
}

Correspondence should be addressed to Srinivasa Rao Kola; srinu.iitkgp@gmail.com

Received 28 April 2018; Accepted 18 August 2018; Published 20 September 2018

Academic Editor: Ali R. Ashrafi

Copyright ( 2018 Srinivasa Rao Kola et al. This is an open access article distributed under the Creative Commons Attribution License, which permits unrestricted use, distribution, and reproduction in any medium, provided the original work is properly cited.

An $L(2,1)$-coloring of a simple connected graph $G$ is an assignment $f$ of nonnegative integers to the vertices of $G$ such that $\mid f(u)$ $f(v) \mid \geqslant 2$ if $d(u, v)=1$ and $|f(u)-f(v)| \geqslant 1$ if $d(u, v)=2$ for all $u, v \in V(G)$, where $d(u, v)$ denotes the distance between $u$ and $v$ in $G$. The span of $f$ is the maximum color assigned by $f$. The span of a graph $G$, denoted by $\lambda(G)$, is the minimum of span over all $L(2,1)$-colorings on $G$. An $L(2,1)$-coloring of $G$ with span $\lambda(G)$ is called a span coloring of $G$. An $L(2,1)$-coloring $f$ is said to be irreducible if there exists no $L(2,1)$-coloring g such that $g(u) \leqslant f(u)$ for all $u \in V(G)$ and $g(v)<f(v)$ for some $v \in V(G)$. If $f$ is an $L(2,1)$-coloring with span $k$, then $h \in\{0,1,2, \ldots, k\}$ is a hole if there is no $v \in V(G)$ such that $f(v)=h$. The maximum number of holes over all irreducible span colorings of $G$ is denoted by $H_{\lambda}(G)$. A tree $T$ with maximum degree $\Delta$ having span $\Delta+1$ is referred to as Type-I tree; otherwise it is Type-II. In this paper, we give a method to construct infinitely many trees with at least one hole from a one-hole tree and infinitely many two-hole trees from a two-hole tree. Also, using the method, we construct infinitely many Type-II trees with maximum number of holes one and two. Further, we give a sufficient condition for a Type-II tree with maximum number of holes zero.

\section{Introduction}

The channel assignment problem is the problem of assigning frequencies to transmitters in some optimal manner. In 1992, Griggs and Yeh [1] have introduced the concept of $L(2,1)$ coloring as a variation of channel assignment problem. The distance between two vertices $u$ and $v$ in a graph $G$, denoted by $d(u, v)$, is defined as the length of a shortest path between $u$ and $v$ in $G$. An $L(2,1)$-coloring of a graph $G$ is an assignment $f: V(G) \longrightarrow\{0,1,2, \ldots, k\}$ such that, for every $u, v$ in $V(G)$, $|f(u)-f(v)| \geqslant 2$ if $u$ and $v$ are adjacent and $|f(u)-f(v)| \geqslant 1$ if $u$ and $v$ are at distance 2 . The nonnegative integers assigned to the vertices are also called colors. The span of $f$, denoted by span $f$, is $\max \{f(v): v \in V(G)\}$. The span of $G$, denoted by $\lambda(G)$, is $\min \{$ span $f: f$ is an $L(2,1)$-coloring of $G\}$. An $L(2,1)$-coloring with span $\lambda(G)$ is called a span coloring. A tree is a connected acyclic graph. In the introductory paper, Griggs and Yeh [1] proved that $\lambda\left(P_{n}\right)=4$ for $n \geqslant 5 ; \lambda(T)$ is either $\Delta+1$ or $\Delta+2$ for any tree $T$ with maximum degree $\Delta$. We refer to a tree as Type-I if $\lambda(T)=\Delta+1$; otherwise it is Type-II. In a graph $G$ with maximum degree $\Delta$, we refer to a vertex $v$ as a major vertex if its degree is $\Delta$; otherwise $v$ is a minor vertex. Wang [2] has proved that a tree with no pair of major vertices at distances 1,2 , and 4 is Type-I. Zhai et al. [3] have improved the above condition as a tree with no pair of major vertices at distances 2 and 4 is Type-I. Mandal and Panigrahi [4] have proved that $\lambda(T)=\Delta+1$ if $T$ has at most one pair of major vertices at distance either 2 or 4 and all other pairs are at distance at least 7. Wood and Jacob [5] have given a complete characterization of the $L(2,1)$-span of trees up to twenty vertices.

Fishburn and Roberts [6] have introduced the concept of no-hole $L(2,1)$-coloring of a graph. If $f$ is an $L(2,1)$ coloring of a graph $G$ with span $k$, then an integer $h \in$ $\{0,1,2, \ldots, k\}$ is called a hole in $f$ if there is no vertex $v$ in $G$ such that $f(v)=h$. An $L(2,1)$-coloring with no hole is called a no-hole coloring of G. Fishburn et al. [7] have introduced the concept of irreducibility of $L(2,1)$-coloring. An $L(2,1)$-coloring of a graph $G$ is reducible if there exists another $L(2,1)$-coloring $g$ of $G$ such that $g(u) \leqslant f(u)$ for all vertices $u$ in $G$ and there exists a vertex $v$ in $G$ such that $g(v)<f(v)$. If $f$ is not reducible then it is called irreducible. 
An irreducible no-hole coloring is referred to as inh-coloring. A graph is inh-colorable if there exists an inh-coloring. For an inh-colorable graph $G$, the lower inh-span or simply inhspan of $G$, denoted by $\lambda_{i n h}(G)$, is defined as $\lambda_{i n h}(G)=$ $\min \{\operatorname{span} f: f$ is an inh-coloring of $G\}$. Fishburn et al. [7] have proved that paths, cycles, and trees are inh-colorable except $C_{3}, C_{4}$, and stars. In addition to that, they showed that $\Delta+1 \leqslant \lambda_{i n h}(T) \leqslant \Delta+2$ where $T$ is any nonstar tree. Laskar et al. [8] have proved that any nonstar tree $T$ is inhcolorable and $\lambda_{\text {inh }}(T)=\lambda(T)$. The maximum number of holes over all irreducible span colorings of $G$ is denoted by $H_{\lambda}(G)$. Laskar and Eyabi [9] have determined the exact values for maximum number of holes for paths, cycles, stars, and complete bipartite graphs as $2,2,1$, and 1, respectively, and conjectured that, for any tree $T, H_{\lambda}(T)=2$ if and only if $T$ is a path $P_{n}, n>4$. S. R. Kola et al. [10] have disproved the conjecture by giving a two-hole irreducible span coloring for a Type-II tree other than path.

In this article, we give a method of construction of infinitely many two-hole trees from a two-hole tree and infinitely many trees with at least one hole from a onehole tree. Also, we find maximum number of holes for some Type-II trees given by Wood and Jacob [5] and obtain infinitely many Type-II trees of holes one and two by applying the method of construction. Further, we give a sufficient condition for a zero-hole Type-II tree.

\section{Construction of Trees with Maximum Number of Holes One and Two}

We start this section with a lemma which gives the possible colors to the major vertices in a two-hole span coloring of a Type-II tree.

Lemma 1. In any two-hole span coloring of a Type-II tree T with $\Delta \geqslant 3$, all major vertices receive either the same color or the colors from any one of the sets $\{0,2\},\{0, \Delta+2\}$, or $\{\Delta, \Delta+2\}$.

Proof. Let $f$ be a two-hole span coloring of a Type-II tree $T$. Suppose that $v_{1}$ and $v_{2}$ are major vertices such that $f\left(v_{1}\right) \neq$ $f\left(v_{2}\right)$. First, we prove that $\left\{f\left(v_{1}\right), f\left(v_{2}\right)\right\}=\{0,2\}$ or $\{0, \Delta+2\}$ or $\{\Delta, \Delta+2\}$. Let $f\left(v_{1}\right)=l$ and $f\left(v_{2}\right)=l^{\prime}$. Without loss of generality, we assume that $0 \leqslant l<l^{\prime} \leqslant \Delta+2$. If $l=0$, then the color 1 must be one of the two holes in $f$. If $l^{\prime} \neq \Delta+2$, then $l^{\prime}-1$ and $l^{\prime}+1$ are the holes. Since $l^{\prime}+1$ cannot be $1, l^{\prime}-1$ is 1 which implies $l^{\prime}=2$. If $l \neq 0$, then $l-1$ and $l+1$ are the holes in $f$. If $l^{\prime} \neq \Delta+2$, then $l^{\prime}-1$ and $l^{\prime}+1$ are the holes which are not possible as $l \neq l^{\prime}$. If $l^{\prime}=\Delta+2$, then $\Delta+1$ must be one of the holes in $f$. Since $l-1$ cannot be $\Delta+1, l+1$ is $\Delta+1$ which implies $l=\Delta$.

If $\left\{f\left(v_{1}\right), f\left(v_{2}\right)\right\}=\{0,2\}$, then 1 and 3 are the holes. If any major vertex $v$ receives a color $l$ other than 0 and 2, then the neighbors of $v$ cannot get the colors 1 and 3 and at least one of $l-1$ and $l+1$ (if $l=\Delta+2$, then $l-1$ ). This is not possible as we need $\Delta+1$ number of colors to color a major vertex and its neighbors. Similarly, other cases can be proved.

The following lemma is a direct implication of Lemma 1.
Lemma 2. If $f$ is a two-hole span coloring of a Type-II tree $T$ having two major vertices at distance less than or equal to two, then the set of holes in $f$ is $\{1,3\},\{1, \Delta+1\}$, or $\{\Delta-1, \Delta+1\}$.

When we say connecting two trees, we mean adding an edge between them. Corresponding to the possibilities of holes given in Lemma 2, we give a list of trees which can be connected to a two-hole tree having two major vertices at distance less than or equal to two, to obtain infinitely many two-hole trees. Later, we give a list of trees which can be connected to a one-hole tree to get infinitely many one-hole trees.

Theorem 3. If $T$ is a tree with maximum number of holes two and having at least two major vertices at distance at most two, then there are infinitely many trees with maximum number of holes two and with maximum degree $\Delta$ same as that of $T$.

Proof. Let $f$ be an irreducible span coloring of $T$ with two holes. Then by Lemma 2 , the set of holes in $f$ is $\{1,3\}$ or $\{1, \Delta+1\}$ or $\{\Delta-1, \Delta+1\}$. Now, we give a method to construct trees from $T$ using the coloring $f$ and holes in $f$. For all the three possibilities of holes, we give a list of trees which can be connected to $T$ to get a bigger tree with maximum number of holes two. Suppose 1 and 3 are the holes in $f$. We use Table 1 for construction.

Let $u$ be a vertex of the tree $T$ and $c$ be the color received by $u$. Now depending on the colors of the neighbors of $u$, to preserve $L(2,1)$-coloring, we connect the trees (one at a time) given in Table 1 by adding an edge between $u$ of $T$ and the vertex colored $k$ of tree in the table. Note that $0 \leqslant k \leqslant \Delta+2$ and the color $k$ is not equal to any of the colors $c-1, c, c+1$, 1 , and 3 and not assigned to any neighbor of $u$. To maintain irreducibility, we use the condition given in the last column of the table. It is easy to see that, after every step, we get a tree $T^{\prime}$ with maximum degree same as that of $T$ and a twohole irreducible span coloring of $T^{\prime}$. Also, it is clear that $T$ is a subtree of $T^{\prime}$. Since connecting a tree to any pendant vertex is always possible, we get infinitely many trees.

Suppose 1 and $\Delta+1$ are the holes in $f$. Construction is similar to the previous case using trees in Table 2.

Suppose $\Delta-1$ and $\Delta+1$ are the holes in $f$. We use trees in Table 3 for construction.

Theorem 4. If $T$ is a tree with $H_{\lambda}(T)=1$, then there exist infinitely many trees containing $T$ and with maximum number of holes at least 1 .

Proof. Here, we start with a one-hole irreducible $L(2,1)$ span coloring of $T$ having hole $h$. The construction of infinitely many trees is similar to that in Theorem 3 and using Table 4 . Since after every step we get a tree $T^{\prime}$ with one-hole irreducible span coloring, $H_{\lambda}\left(T^{\prime}\right) \geqslant 1$.

Theorem 5. If $T$ is a tree with $H_{\lambda}(T)=1$ and $T$ has no twohole span coloring, then there exist infinitely many trees with maximum number of holes one and containing $T$.

Proof. Since $T$ has no two-hole span coloring, any tree containing $T$ having same maximum degree as that of $T$ 
TABLE 1: Trees connectable to a vertex $u$ colored $c$ in a two-hole tree with 1 and 3 as holes.

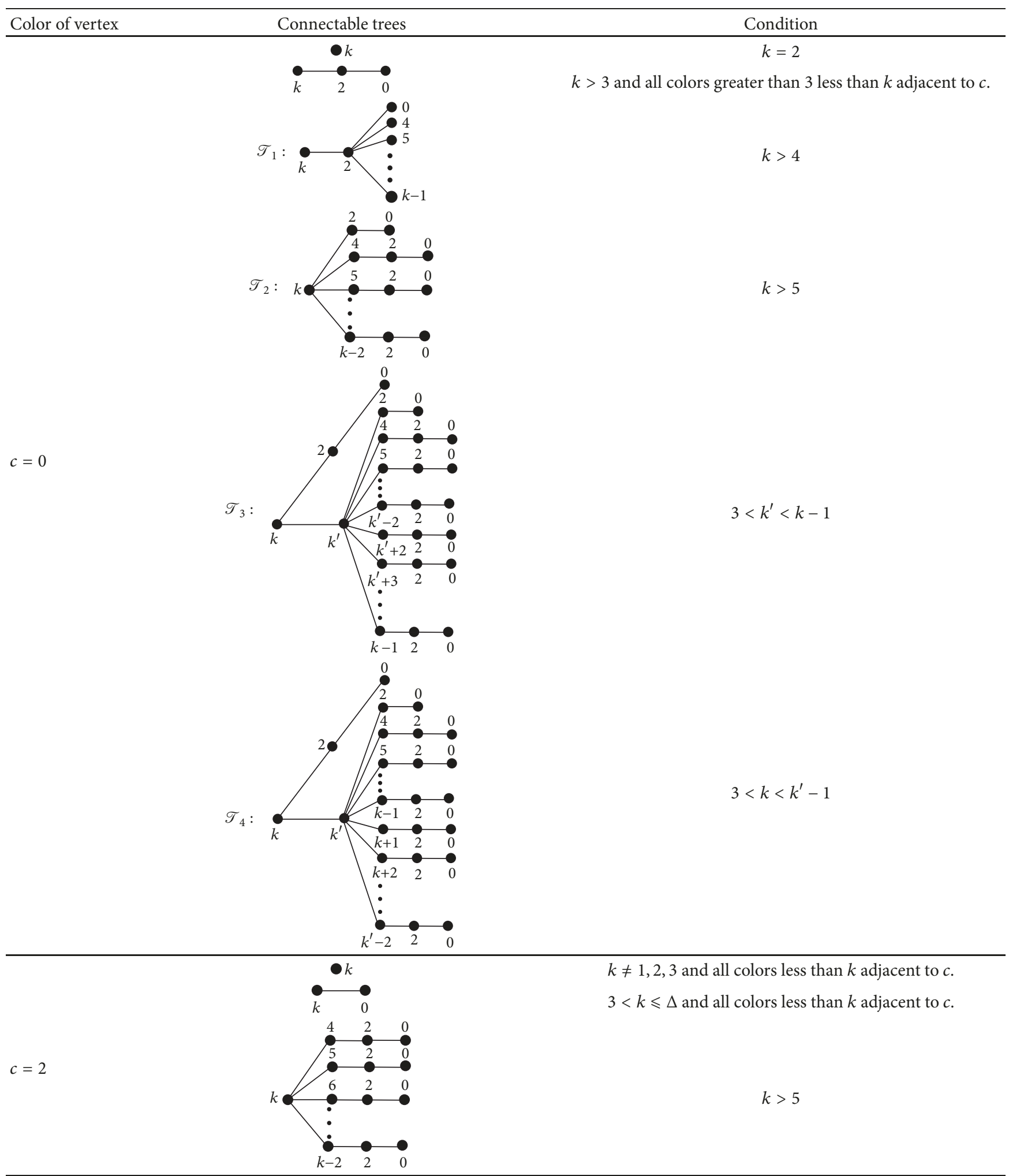


TABle 1: Continued.

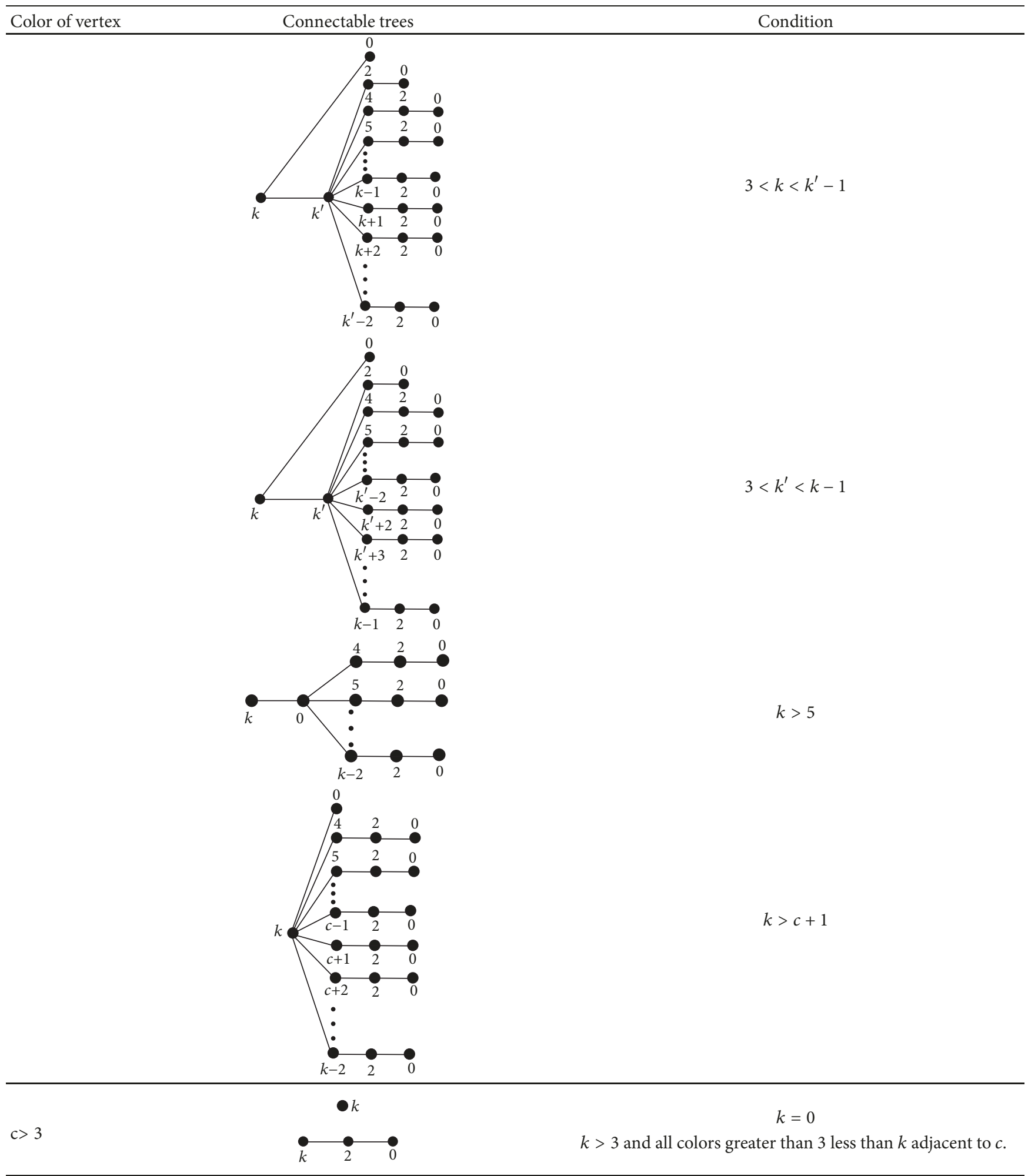


TABLe 1: Continued.

Color of vertex

cannot have a two-hole span coloring. Therefore, every tree obtained from $T$ using Theorem 4 has maximum number of holes one.

Corollary 6. If $T$ is a Type-I tree and $H_{\lambda}(T)=1$, then there exist infinitely many trees with maximum number of holes one and containing $T$.

\section{Maximum Number of Holes in Some Type-II Trees}

Recall that, in a graph $G$ with maximum degree $\Delta$, we refer a vertex $v$ as a major vertex if its degree is $\Delta$. Otherwise $v$ is a minor vertex. Wood and Jacob [5] have given some sufficient conditions for a tree to be Type-II. We consider some of their sufficient conditions as below.

Theorem 7 (see [5]). A tree containing any of the following subtrees is Type-II provided the maximum degrees of the subtree and the tree are the same $\Delta$.

(I) $T_{1}$ : a tree with an induced $P_{3}$ consisting of three major vertices.

(II) $T_{2}$ : a tree with a minor vertex $w$ and at least 3 major vertices adjacent to $w$.

(III) $T_{3}$ : a tree with a major vertex $w$ and at least $\Delta-1$ major vertices at distance two from $w$, and $T_{2}$ is not a subtree of the tree.
(IV) $T_{4}$ : a tree with a vertex $w$ adjacent to $\Delta-2$ vertices $w_{1}, w_{2}, \ldots, w_{\Delta-2}$ and two neighbors $v_{i}, v_{i}^{\prime}$ of each $w_{i}$, $1 \leqslant i \leqslant \Delta-2$ are major.

Since the above trees can be as small as possible, we consider the degrees of minor vertices as minimum as possible. Now, we find the maximum number of holes for the trees $T_{1}, T_{2}, T_{3}$, and $T_{4}$. For any tree $T$ with maximum degree $\Delta$, it is clear that $H_{\lambda}(T) \leqslant 2$. First, we show that $H_{\lambda}\left(T_{i}\right) \leqslant 1, i=1,2,4$. Also, $H_{\lambda}\left(T_{2}\right)=0$ if $T_{2}$ has a vertex adjacent to at least four major vertices. Further, we give a two-hole $L(2,1)$-irreducible span coloring of $T_{3}$ if it has exactly $\Delta-1$ major vertices at distance two from a major vertex and we show that $H_{\lambda}\left(T_{3}\right) \leqslant$ 1 , if $T_{3}$ has exactly $\Delta$ major vertices at distance two from a major vertex. Later, we show that these upper bounds are the exact values by defining $L(2,1)$-irreducible span colorings with appropriate holes. Now onwards, unless we mention, tree refers to Type-II tree. In figures, we use symbol $\boldsymbol{\Delta}$ to denote a major vertex.

Theorem 8. For the trees $T_{i}, i=1,2,4, H_{\lambda}\left(T_{i}\right) \leqslant 1$.

Proof. Let $v_{1}, v_{2}$, and $v_{3}$ be the major vertices of $T_{1}$. Since $v_{1}, v_{2}$, and $v_{3}$ receive three different colors in any $L(2,1)$ coloring, by Lemma $1, T_{1}$ cannot have a two-hole irreducible span coloring. Similarly, we can prove that $H_{\lambda}\left(T_{2}\right) \leqslant 1$.

Now, we consider $T_{4}$ with labelling as in Figure 1.

Suppose that $f$ is a two-hole irreducible span coloring of $T_{4}$. Then by Lemma 1 , all major vertices of $T_{4}$ receive colors from $\{0,2\}$ or $\{0, \Delta+2\}$ or $\{\Delta, \Delta+2\}$. Suppose the major vertices receive 0 and 2 . Then 1 and 3 are holes. Without loss 
TABLE 2: Trees connectable to a vertex $u$ colored $c$ in a two-hole tree with 1 and $\Delta+1$ as holes.

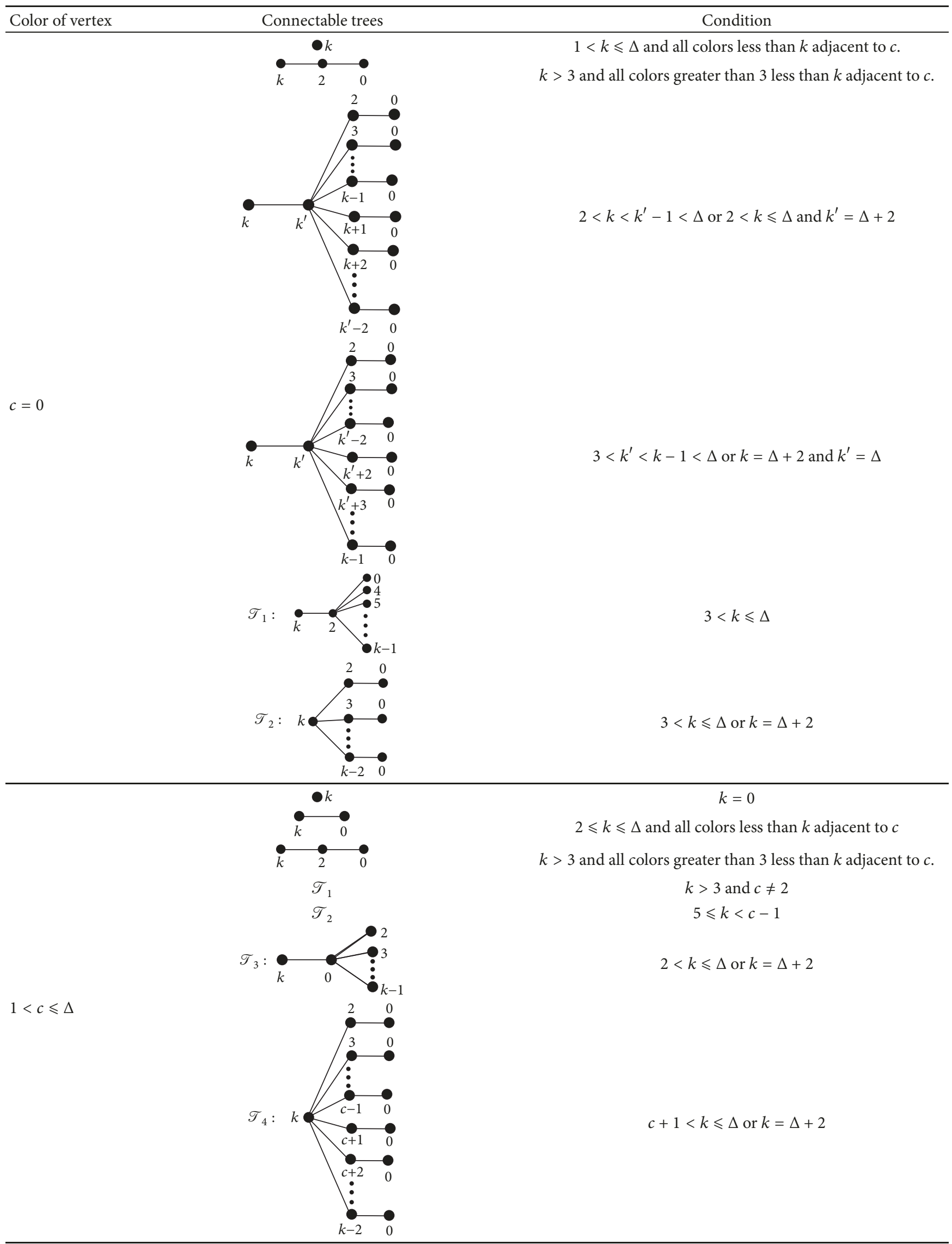


TABLE 2: Continued.

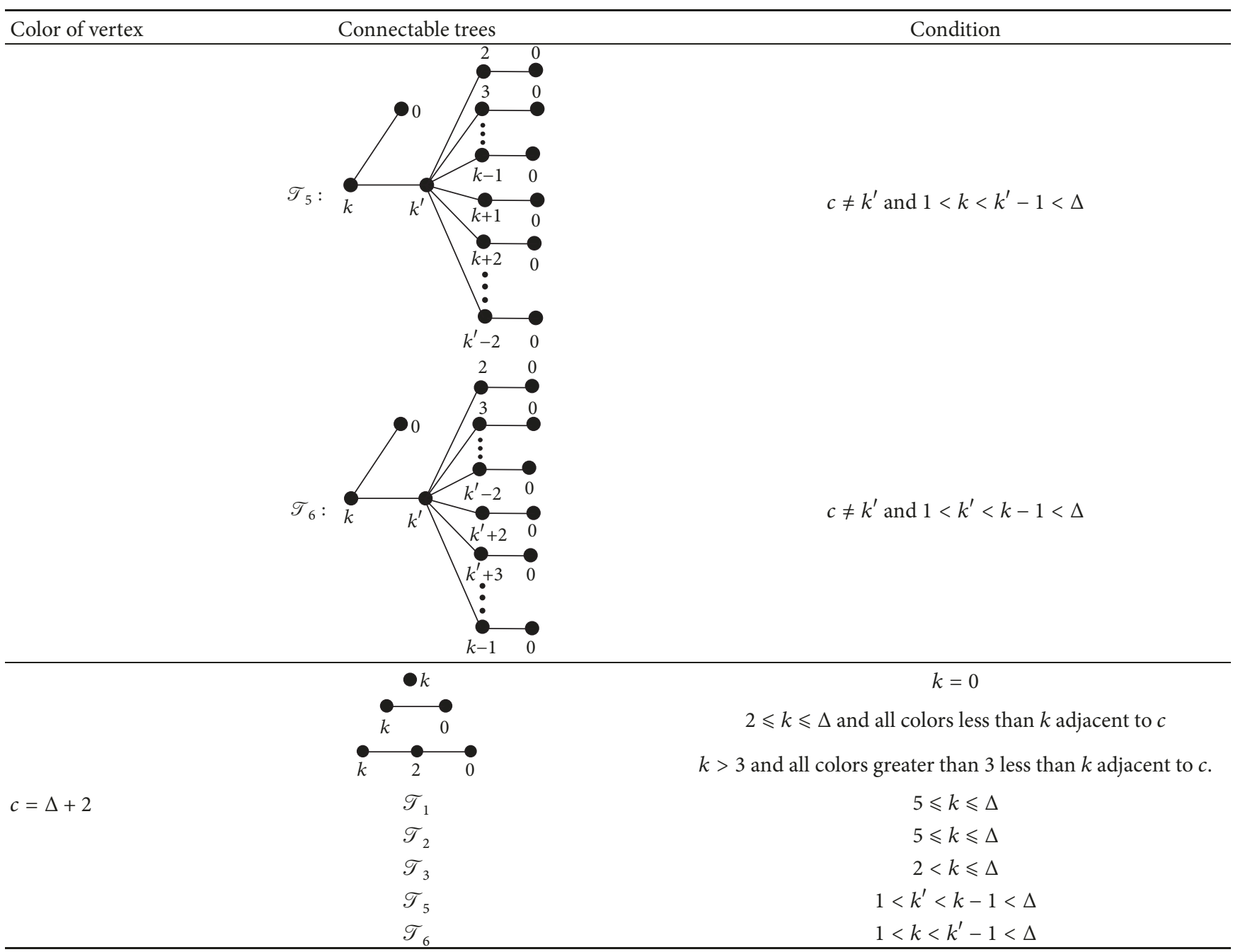

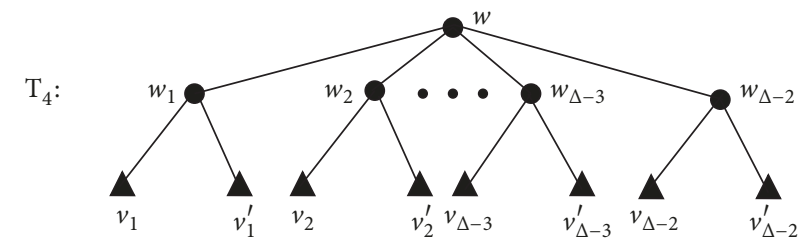

Figure 1: The tree $T_{4}$ as in Theorem 7 .

of generality, we assume that $f\left(v_{i}\right)=0$ and $f\left(v_{i}^{\prime}\right)=2$. Now, one of the pendant vertices adjacent to $v_{i}$ must receive a color grater than 3 which reduces to 3 giving a contradiction to the fact that $f$ is irreducible. Similarly, we can prove the other two cases. Therefore, $H_{\lambda}\left(T_{4}\right) \leqslant 1$.

Theorem 9. If at least four major vertices are adjacent to $w$ in $T_{2}$, then $H_{\lambda}\left(T_{2}\right)=0$.

Proof. Recall that $T_{2}$ is a tree with a vertex $w$ adjacent to at least three major vertices. Let $v_{1}, v_{2}, v_{3}$, and $v_{4}$ be four major vertices adjacent to $w$ in $T_{2}$. Suppose that it has a one-hole irreducible $L(2,1)$-span coloring $f$. Let $l_{1}, l_{2}, l_{3}$, and $l_{4}$ be the colors received by $v_{1}, v_{2}, v_{3}$, and $v_{4}$, respectively. Without loss of generality, we assume that $0 \leqslant l_{1}<l_{2}<l_{3}<l_{4} \leqslant \Delta+2$. If $l_{1} \neq 0$, then except $l_{1}-1$ and $l_{1}+1$ all other colors are used to the neighbors of $v_{1}$. Also, except $l_{3}-1$ and $l_{3}+1$, all other colors are used to the neighbors of $v_{3}$. Since $l_{1}-1, l_{1}+$ $1, l_{3}-1$, and $l_{3}+1$ are four different colors, $f$ cannot have a hole which is a contradiction. So, $l_{1}=0$. Since $f$ is one-hole coloring, the colors $l_{2}-1, l_{2}+1, l_{3}-1$, and $l_{3}+1$ cannot be four different colors and hence $l_{2}+1=l_{3}-1$ is the hole. Now, a pendant neighbor of $v_{1}$ receives $l_{3}$ which reduces to the hole $l_{3}-1$ giving a contradiction to the fact that $f$ is irreducible.

S. R. Kola et al. [10] have disproved the conjecture given by Laskar and Eyabi [9] by giving two-hole irreducible span 
TABLE 3: Trees connectable to a vertex $u$ colored $c$ in a two-hole tree with $\Delta-1$ and $\Delta+1$ as holes.

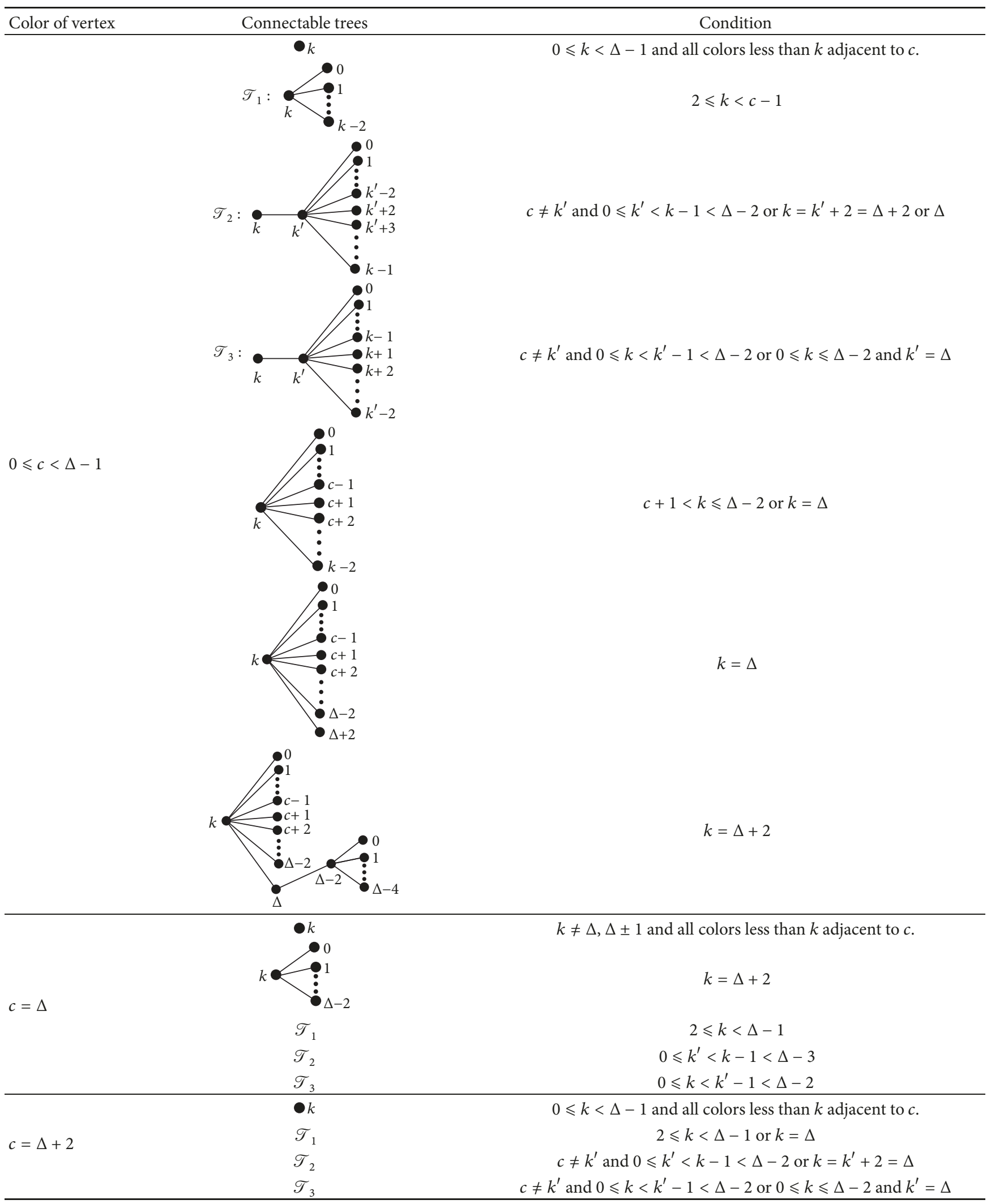


TABLE 4: Trees connectable for a vertex colored $c$ in a one-hole tree with hole $h$.

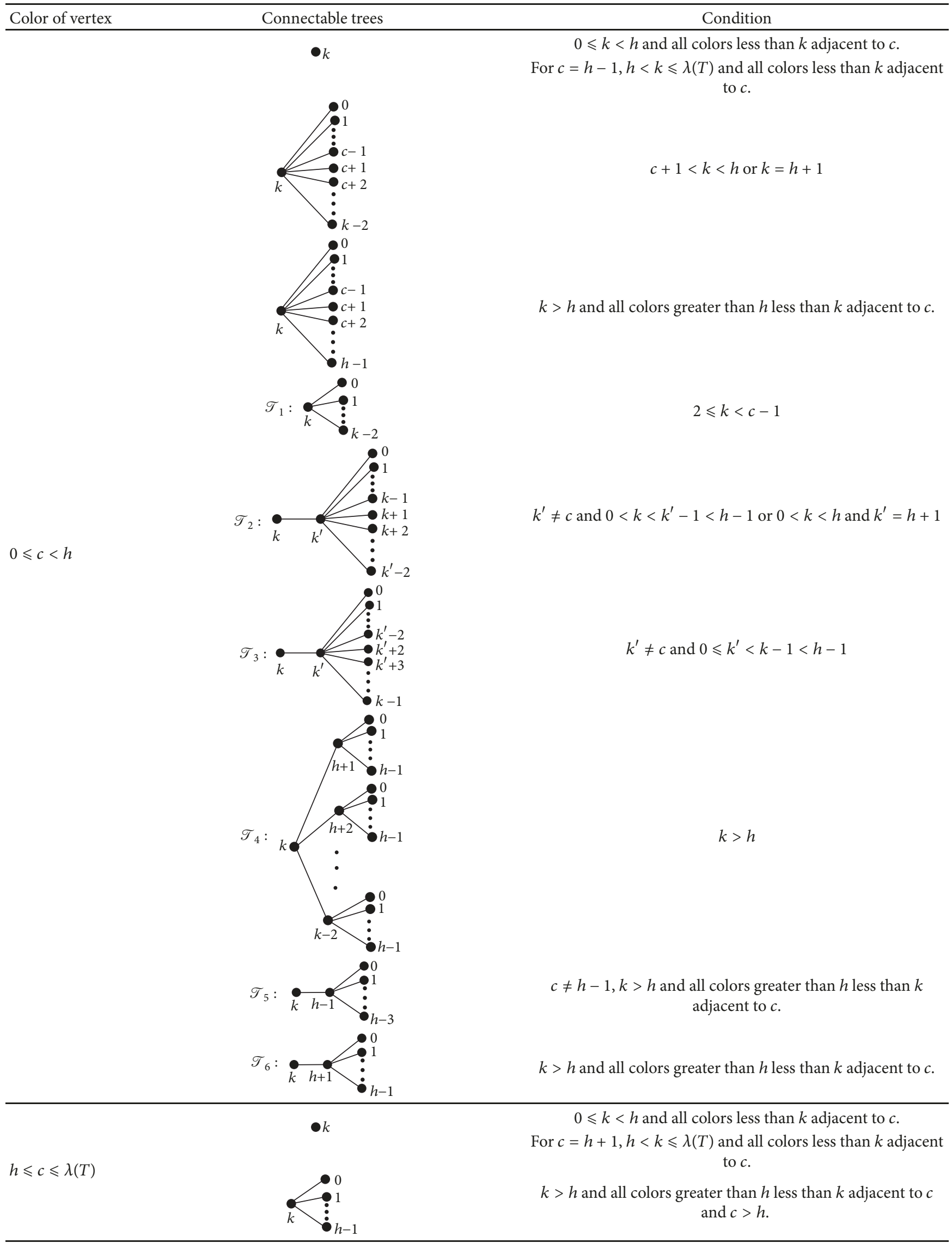


TABLE 4: Continued.

\begin{tabular}{|c|c|c|}
\hline Color of vertex & Connectable trees & Condition \\
\hline & & $h<c<k-1$ \\
\hline & $\mathscr{T}_{1}$ & $2 \leqslant k<h$ or $k=h+1$ \\
\hline & $\mathscr{T}_{2}$ & $0<k<k^{\prime}-1<h-1$ or $0<k<h$ and $k^{\prime}=h+1$ \\
\hline & $\mathscr{T}_{3}$ & $0 \leqslant k^{\prime}<k-1<h-1$ \\
\hline & $\mathscr{T}_{4}$ & $h+2<k<c-1$ \\
\hline & $\mathscr{T}_{5}$ & $k>h$ and all colors greater than $h$ less than $k$ adjacent to $c$. \\
\hline & $\mathscr{T}_{6}$ & $\begin{array}{c}c \neq h+1, k>h \text { and all colors greater than } h \text { less than } k \\
\text { adjacent to } c .\end{array}$ \\
\hline
\end{tabular}

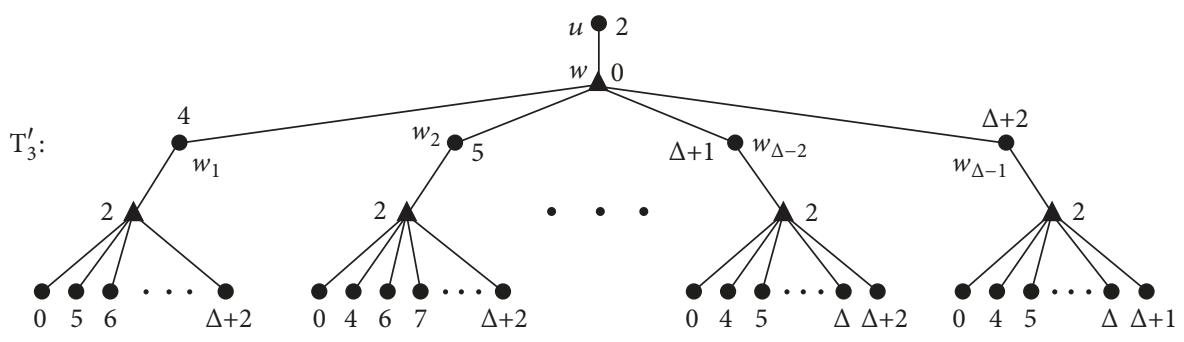

FIgURE 2: Irreducible $L(2,1)$-span coloring of $T_{3}$ with 1 and 3 as holes.

colorings for Type-II trees of maximum degrees three and four. Following theorem gives a two-hole irreducible span coloring for a tree with maximum degree $\Delta$ which is also a counterexample for the conjecture.

Theorem 10. If exactly $\Delta-1$ major vertices are at distance two from the major vertex $w$ in $T_{3}$, then $H_{\lambda}\left(T_{3}\right)=2$.

Proof. Let $T_{3}^{\prime}$ be the tree $T_{3}$ with exactly $\Delta-1$ major vertices at distance two to $w$. It is easy to see that the $L(2,1)$-span coloring of the tree $T_{3}^{\prime}$ given in Figure 2 is irreducible with 1 and 3 as holes.

Theorem 11. If $T_{3}^{\prime \prime}$ is the tree $T_{3}$ with exactly $\Delta$ major vertices at distance two to $w$, then $H_{\lambda}\left(T_{3}^{\prime \prime}\right) \leqslant 1$.

Proof. We consider $T_{3}^{\prime \prime}$ with labelling as in Figure 3.

Suppose that $T_{3}^{\prime \prime}$ has an $L(2,1)$-span coloring $f$ with two holes. Then by Lemma 1 , all major vertices of $T_{3}^{\prime \prime}$ receive colors from $\{0,2\}$ or $\{0, \Delta+2\}$ or $\{\Delta, \Delta+2\}$. Suppose that $f$ assigns 0 and 2 to the major vertices. Since $\left\{f(w), f\left(v_{i}\right)\right\}=$
$\{0,2\}, 1 \leqslant i \leqslant \Delta$, it is not possible to color all $w_{i}$ s as the four colors $0,1,2$, and 3 cannot be assigned. Therefore, in this case, two-hole span coloring is not possible for $T_{3}^{\prime \prime}$. Similarly, we can prove the other two cases. Hence, $H_{\lambda}\left(T_{3}^{\prime \prime}\right) \leqslant 1$.

Let $T_{2}^{\prime}$ be the tree $T_{2}$ with exactly three major vertices are adjacent to a vertex.

Theorem 12. For the trees $T_{1}, T_{2}^{\prime}, T_{3}^{\prime \prime}$, and $T_{4}$, the maximum number of holes is one.

Proof. It is easy to see that the colorings of $T_{1}, T_{2}^{\prime}, T_{3}^{\prime \prime}$, and $T_{4}$ given in Figures 4, 5, 6, and 7, respectively, are irreducible $L(2,1)$-span colorings with hole $\Delta$.

\section{Infinitely Many Trees with Holes 0,1 , and 2}

Recall that $T_{2}^{\prime}$ is the tree $T_{2}$ with exactly three major vertices adjacent to a vertex and $T_{3}^{\prime \prime}$ is the tree $T_{3}$ with exactly $\Delta$ major vertices at distance two from a major vertex. Let $T_{2}^{\prime \prime}$ be the tree $T_{2}$ with exactly four major vertices adjacent to the vertex 
$\mathrm{T}_{3}^{\prime \prime}$ :

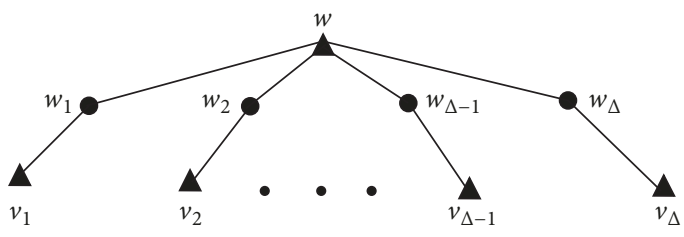

FIGURE 3: The tree $T_{3}$ with exactly $\Delta$ major vertices at distance two to $w$.

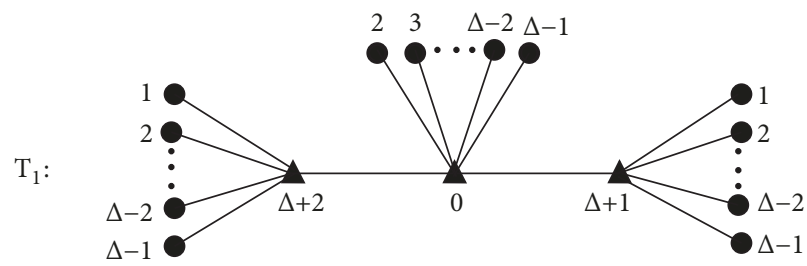

FIgURE 4: Irreducible $L(2,1)$-span coloring of $T_{1}$ with one hole.

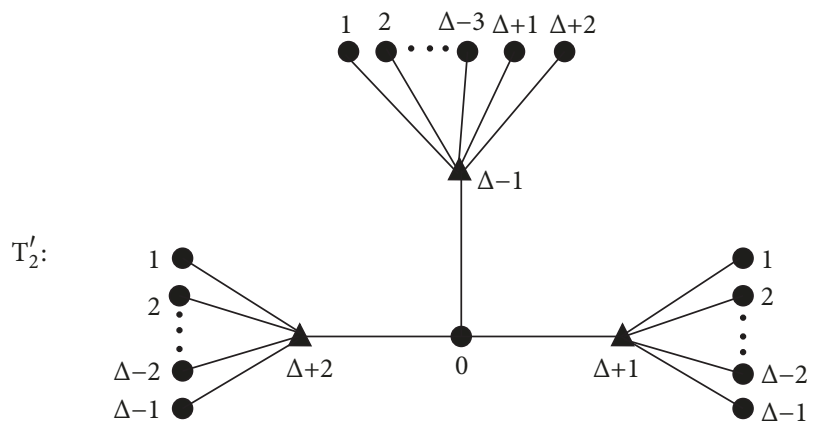

FIGURE 5: Irreducible $L(2,1)$-span coloring of $T_{2}^{\prime}$ with one hole.

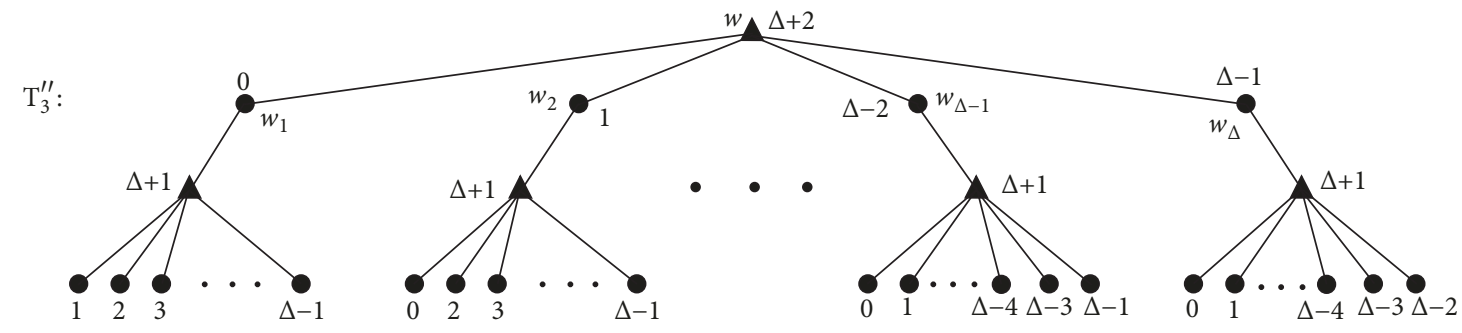

FIgURE 6: Irreducible $L(2,1)$-span coloring of $T_{3}^{\prime \prime}$ with one hole.

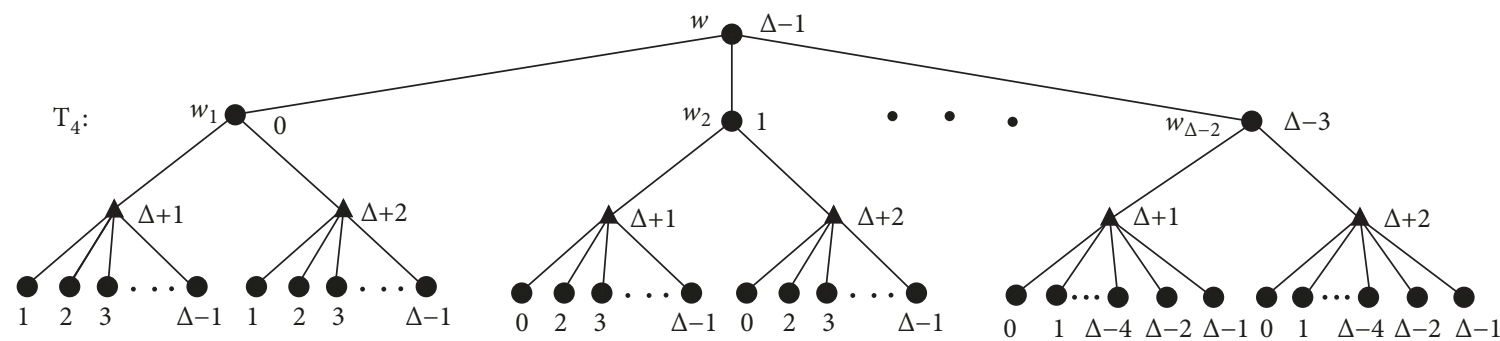

FIGURE 7: Irreducible $L(2,1)$-span coloring of $T_{4}$ with one hole. 


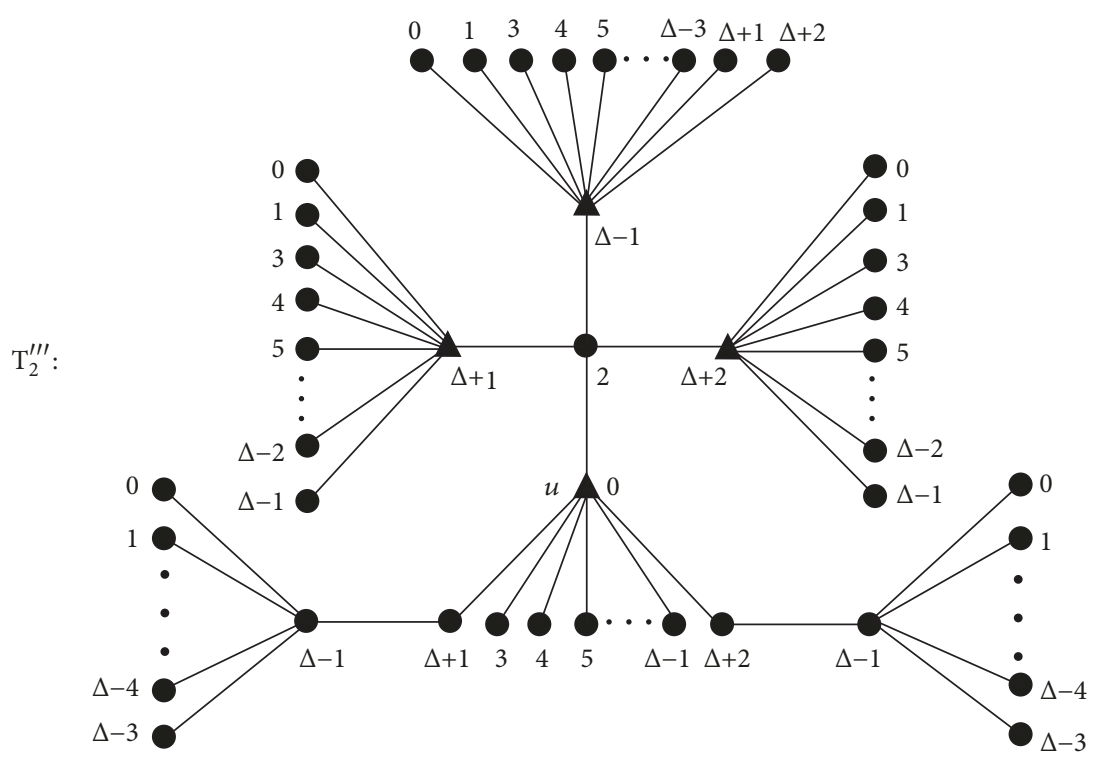

FIGURE 8: Irreducible $L(2,1)$-span coloring of $T_{2}^{\prime \prime \prime}$ with $\Delta$ as hole.

$w$. In this section, we give a sufficient condition for a TypeII tree to be a zero-hole tree. Also, we construct infinitely many trees with maximum number of holes 1 from each of the trees $T_{1}, T_{2}^{\prime}, T_{2}^{\prime \prime}, T_{3}^{\prime \prime}$, and $T_{4}$ and infinitely many two-hole trees containing $T_{3}^{\prime}$.

Theorem 13. If the tree $T_{2}$ with at least five major vertices is a subtree of a tree $T$ with maximum degree same as that of $T_{2}$, then $H_{\lambda}(T)=0$.

Proof. Let $v_{1}, v_{2}, v_{3}, v_{4}$, and $v_{5}$ be five major vertices adjacent to $w$ and receive the colors $l_{1}, l_{2}, l_{3}, l_{4}$, and $l_{5}$, respectively, by a one-hole span coloring $f$ of $T_{2}$. Without loss of generality, we assume that $0 \leqslant l_{1}<l_{2}<l_{3}<l_{4}<l_{5} \leqslant \Delta+2$. As in the proof of Theorem 9 , we get $l_{1}=0$ and $l_{2}+1=l_{3}-1$ is the hole. Since $l_{4}<l_{5} \leqslant \Delta+2$, we have $l_{4} \neq \Delta+2$. Since $l_{4}>l_{3}, l_{3}-1$ must be used to a neighbor of $v_{4}$ which is a contradiction. So, any $L(2,1)$-span coloring of $T_{2}$ with at least five major vertices is a no-hole coloring. Therefore, if a tree $T$ contains $T_{2}$ with at least five major vertices and with maximum degree same as that of $T_{2}$, then $H_{\lambda}(T)=0$.

Theorem 14. There are infinitely many trees with maximum number of holes one and containing each of the trees $T_{1}, T_{2}^{\prime}$, $T_{2}^{\prime \prime}, T_{3}^{\prime \prime}$, and $T_{4}$.

Proof. First, we prove that $T_{1}, T_{2}^{\prime}, T_{2}^{\prime \prime}, T_{3}^{\prime \prime}$, and $T_{4}$ cannot have two-hole span coloring. From Theorems 8 and 11, it is clear that $T_{1}, T_{2}^{\prime}, T_{2}^{\prime \prime}$, and $T_{3}^{\prime \prime}$ cannot have two-hole span colorings.

Next, we prove that $T_{4}$ cannot have a two-hole span coloring. We consider $T_{4}$ with the labelling as in Theorem 12. Suppose that $T_{4}$ has an $L(2,1)$-span coloring $f$ with two holes. By Lemma 1 , any major vertex of $T_{4}$ receives the color from $\{0,2\},\{0, \Delta+2\}$, or $\{\Delta, \Delta+2\}$. Suppose $f$ assigns 0 and 2 to major vertices. Then 1 and 3 are holes, $\left\{f\left(v_{i}\right), f\left(v_{i}^{\prime}\right)\right\}=\{0,2\}$, and $w_{i}$ cannot receive the colors $0,1,2$, and 3 . Therefore $w_{i} \mathrm{~s}$ receive $\Delta-2$ different colors among $4,5,6, \ldots, \Delta+2(\Delta-1$ in number) and so, one of these colors is not used, say $c$. Since either $c-1$ or $c+1$ (if $c=\Delta+2$ then $c-1$ ) is used to color one of the $w_{i}$ s, c cannot be used to $w$. Since 1 and 3 are holes, there is no color for $w$. Similarly, we can prove the other cases.

Now, to use Theorem 4, we need one-hole irreducible span coloring of $T_{1}, T_{2}^{\prime}, T_{2}^{\prime \prime}, T_{3}^{\prime \prime}$, and $T_{4}$. Since $H_{\lambda}\left(T_{2}^{\prime \prime}\right)$ is 0 , first we construct a tree $T_{2}^{\prime \prime \prime}$ from $T_{2}^{\prime \prime}$ such that $H_{\lambda}\left(T_{2}^{\prime \prime \prime}\right)=1$. We define a one-hole span coloring for $T_{2}^{\prime \prime}$ as in Figure $8\left(T_{2}^{\prime \prime}\right.$ is a subtree of $\left.T_{2}^{\prime \prime \prime}\right)$. Since the colors $\Delta+1$ and $\Delta+2$ received by the vertices adjacent to the vertex $u$ are reducible and there is no other color reducible, we connect star $K_{1, \Delta-2}$ to the vertices to make the colors $\Delta+1$ and $\Delta+2$ irreducible. The tree obtained is $T_{2}^{\prime \prime \prime}$.

Now, using Table 5 obtained from Table 4 corresponding to the hole $h=\Delta$ and using irreducible one-hole span colorings of $T_{1}, T_{2}^{\prime}, T_{3}^{\prime \prime}$, and $T_{4}$ given in Theorem 12, we construct infinitely many one-hole trees containing each of the trees $T_{1}, T_{2}^{\prime}, T_{3}^{\prime \prime}$, and $T_{4}$, respectively. We get infinitely many trees containing $T_{2}^{\prime \prime}$ by using irreducible one-hole coloring of $T_{2}^{\prime \prime \prime}$ given in Figure 8 and using Table 5.

Example 15. In Figure 9, we illustrate the construction of onehole tree as in Theorem 14 for the tree $T_{1}$ with maximum degree $\Delta=7$. The vertex $b_{1}$ in $T_{1}$ has color 4 and its neighbor's color is 8 . In Table 5 , among the trees corresponding to the color $c=4$, the pendant vertex colored 0 is connected first. Later, pendant vertices colored 1 and 2 are connected, respectively. Similarly, some trees are connected to the vertices $b_{2}$, $b_{3}$, and $d_{i}, 1 \leqslant i \leqslant 3$.

Theorem 16. There are infinitely many trees containing $T_{3}^{\prime}$ and with maximum number of holes two.

Proof. The construction of trees is similar to the construction described in Theorem 3. For the construction, we use 
TABLE 5: Trees connectable for a vertex colored $c$ in a one-hole tree with $\Delta$ as the hole.

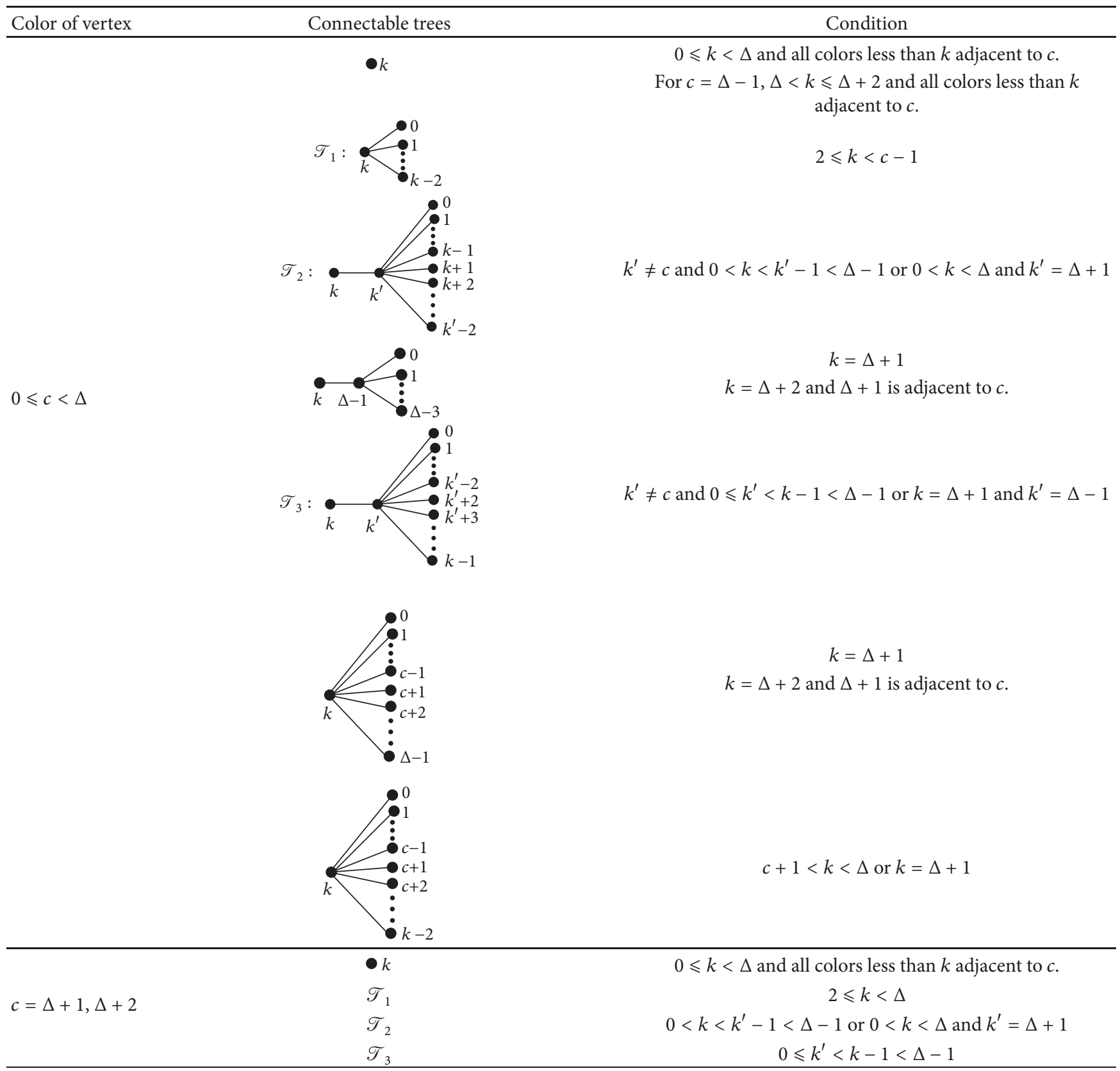

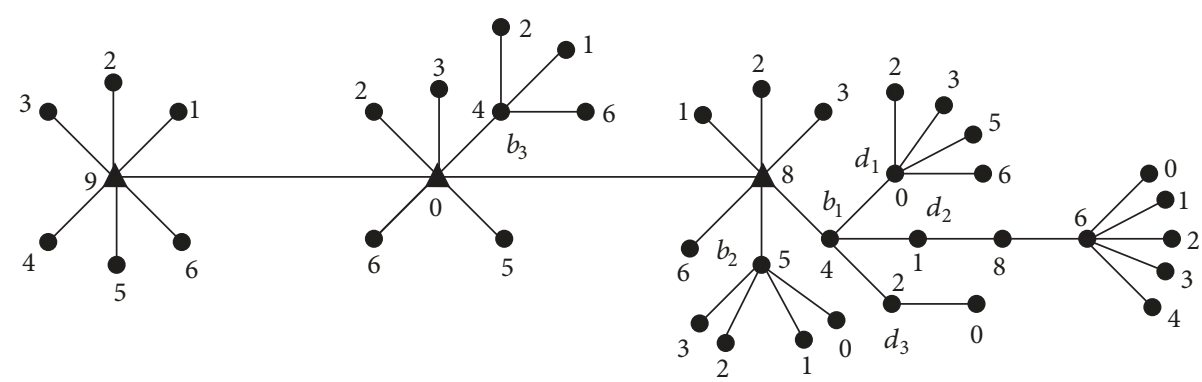

FIGURE 9: A tree with maximum number of holes one constructed from $T_{1}$. 
two-hole irreducible span coloring of $T_{3}^{\prime}$ given in Figure 2 and Table 3.

\section{Data Availability}

The authors confirm that the data supporting the findings of this study are available within the article.

\section{Conflicts of Interest}

The authors declare that they have no conflicts of interest.

\section{References}

[1] J. R. Griggs and R. K. Yeh, "Labeling graphs with a condition at distance 2," SIAM Journal on Discrete Mathematics, vol. 5, no. 4, pp. 586-595, 1992.

[2] W.-F. Wang, "The L(2,1)-labelling of trees," Discrete Applied Mathematics, vol. 154, no. 3, pp. 598-603, 2006.

[3] M.-q. Zhai, C.-h. Lu, and J.-l. Shu, "A note on L(2,1)-labelling of trees," Acta Mathematicae Applicatae Sinica, vol. 28, no. 2, pp. 395-400, 2012.

[4] N. Mandal and P. Panigrahi, "Solutions of some L(2,1)-coloring related open problems," Discussiones Mathematicae Graph Theory, vol. 36, no. 2, pp. 279-297, 2016.

[5] C. A. Wood and J. Jacob, "A complete L(2,1)-span characterization for small trees," AKCE International Journal of Graphs and Combinatorics, vol. 12, no. 1, pp. 26-31, 2015.

[6] P. C. Fishburn and F. S. Roberts, "No-hole L(2,1)-colorings," Discrete Applied Mathematics, vol. 130, no. 3, pp. 513-519, 2003.

[7] P. C. Fishburn, R. C. Laskar, F. S. Roberts, and J. Villalpando, "Parameters of L $(2,1)$-coloring," Manuscript.

[8] R. C. Laskar, G. L. Matthews, B. Novick, and J. Villalpando, "On irreducible no-hole L(2,1)-coloring of trees," Networks. An International Journal, vol. 53, no. 2, pp. 206-211, 2009.

[9] R. Laskar and G. Eyabi, "Holes in L(2,1)-coloring on certain classes of graphs," AKCE International Journal of Graphs and Combinatorics, vol. 6, no. 2, pp. 329-339, 2009.

[10] S. R. Kola, B. Gudla, and N. P.K., "Some classes of trees with maximum number of holes two," AKCE International Journal of Graphs and Combinatorics, 2018. 


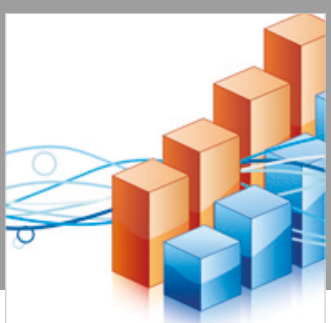

Advances in

Operations Research

\section{-n-m}
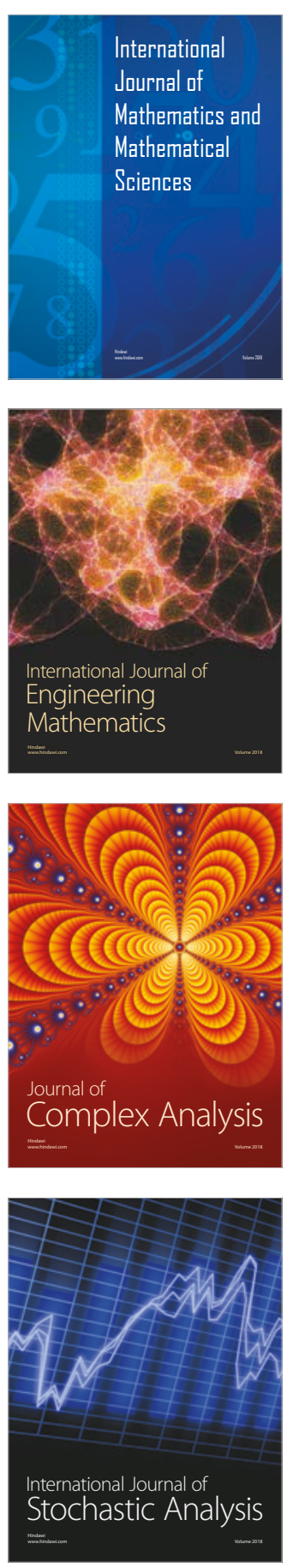
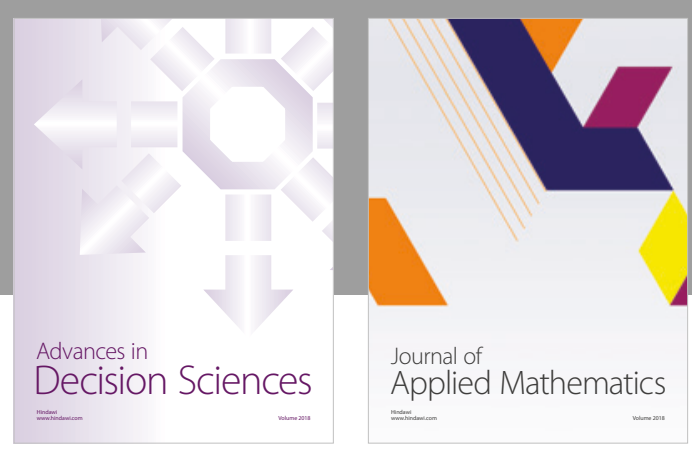

Journal of

Applied Mathematics
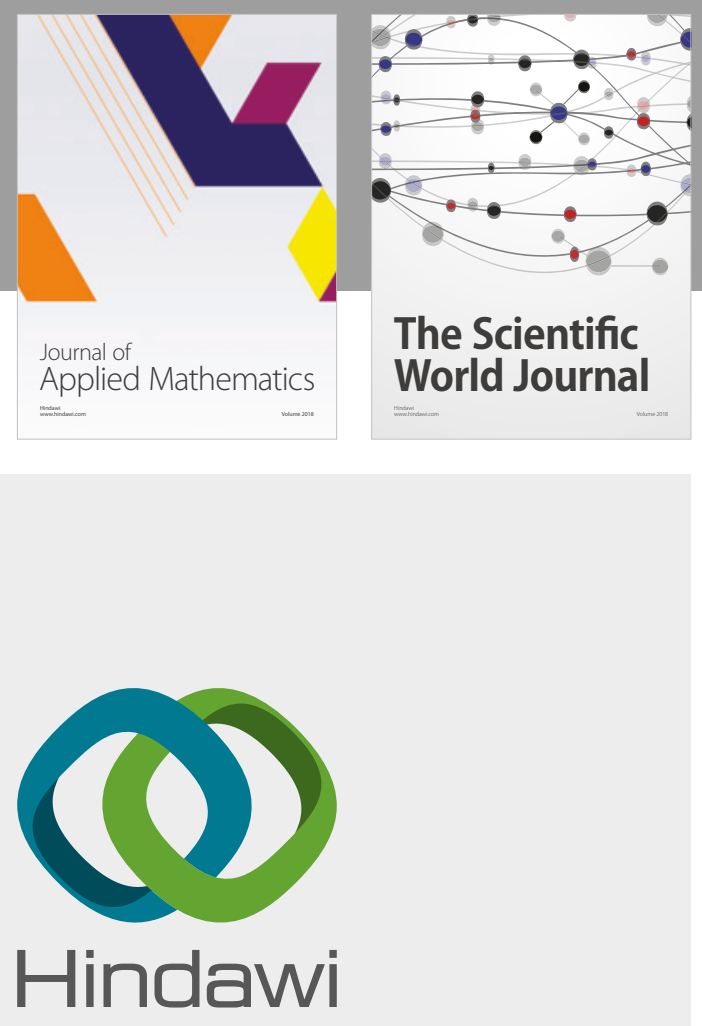

Submit your manuscripts at

www.hindawi.com

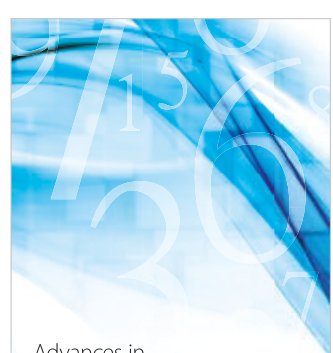

Advances in
Numerical Analysis
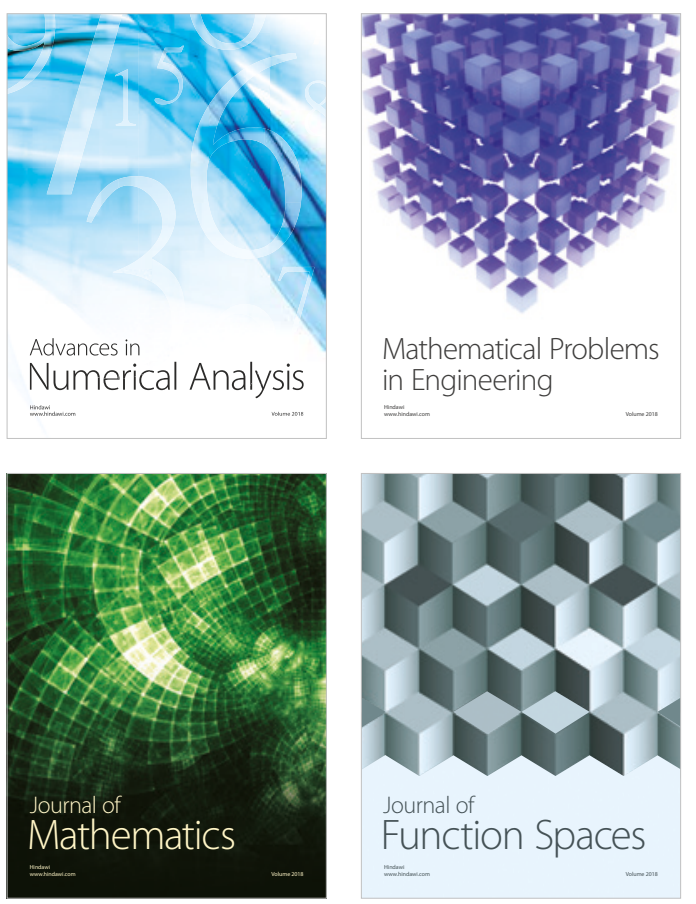

Mathematical Problems in Engineering

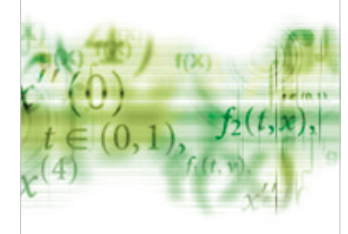

International Journal of

Differential Equations

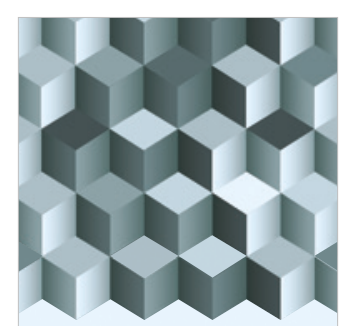

Journal of

Function Spaces

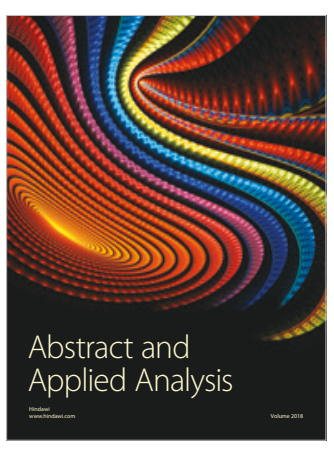

The Scientific

World Journal

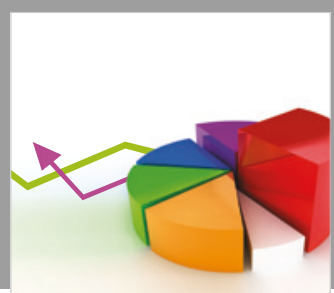

Journal of

Probability and Statistics
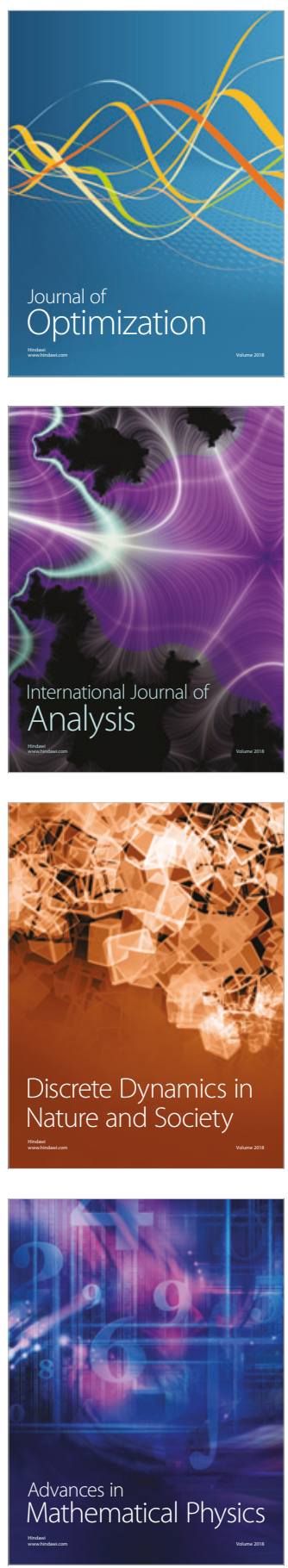